\title{
Non-static plane symmetric massive string magnetized perfect fluid cosmological models in bimetric theory of gravitation
}

\author{
R. C. Sahu, B. Behera ${ }^{1}$ and S. P. Misra ${ }^{2}$ \\ Department of Mathematics, K.S.U.B. College, Bhanjanagar-761126, Odisha, India \\ ${ }^{I}$ Department of Mathematics, N.S.B Mohabidyalaya, Nuvapada, Ganjam, Odisha \\ ${ }^{2}$ Department of Mathematics, Sri Jagannath Mahavidyalaya, Rambha, Ganjam, Odisha
}

\begin{abstract}
In this paper,we investigate the non-static massive string magnetized barotropic perfect fluid cosmological models in Rosen's [Gen.Rel.Grav.Vol.4 (1973) 435] bimetric theory of gravitation. Using the relation isotropic pressure ' $p$ ' directly proportional to energy density ' $\rho$ ' (i.e $p=\epsilon 1 \rho$, where $-1 \leq \epsilon 1 \leq 1)$, dust filled geometric string model and stiff fluid filled model of the universe are obtained. Some physical and geometrical behavior of the exhibited models are also discussed.
\end{abstract}

PACS: 04.50. $+h$

Keywords: Bimetric theory, magnetic field, cosmic string, perfect fluid.

\section{Introduction}

We know that most of the cosmological models based on the theory of Einstein's general relativity contain an initial singularity (the big-bang) from which the universe expands. The singularity can be avoided if the behavior of the matter and radiation is described by the quantum theory. But it is matter of regrettable that not a single author is able to develop a satisfactory physical theory through which singularity free mathematical models of the universe can be constructed because presence of singularity means a break-down of physical laws. Taking the facts into consideration and to get rid of the singularities that occur in general relativity and appear in the big-bang cosmological models, Rosen [1] proposed a new theory of gravitation known as bimetric relativity. In this theory there exists two metric tensors at each point of the space time whose

role is to determine physical situation. The first metric tensor $g_{i j}$ determines the Riemannian geometry of curved space time which plays the same role as in general relativity and it interacts with matter. The back ground metric tensor $\gamma_{i j}$ refers to the geometry of the empty universe and described the inertial forces. It has no

direct physical significance but appears in the field equations. Also it interacts with $g_{i j}$ but not directly with matter. One can regards $\gamma_{i j}$ as giving the geometry that would exist if there were no matter. This theory also satisfies the covariant and equivalence principles. It is also pointed out that this theory agrees with the theory general relativity up to the accuracy of observations made till the date.

The field equations of bimetric theory of gravitation proposed by Rosen [1] are

$$
N_{j}^{i}-\frac{1}{2} \mathrm{~N} \delta_{j}^{i}=
$$

$8 \pi \mathrm{k} T_{j}^{i}$

(1)

where

$N_{j}^{i}=\frac{1}{2} \gamma \mathrm{ab}\left(g^{h i} g_{h j \mid \mathrm{a}}\right)_{\mid \mathrm{b}}$

and

$\mathrm{N}=N_{j}^{i}, \quad(\mathrm{i}, \mathrm{j}=1,2,3,4) ; \quad \mathrm{k}=\sqrt{\frac{\mathrm{g}}{\gamma}}$

together with $\mathrm{g}=$ determinant of $g_{i j}$ and $\gamma=$ determinant of $\gamma_{i j}$.

Here the vertical bar (|) denotes the covariant differentiation with respect to $\gamma_{i j}$ and $T_{j}^{i}$ is the energy momentum tensor of the matter.

In recent days there has been a lot of interest on the study of cosmic strings which are topologically stable objects. These might be found during a phase transition in the early universe i.e. after the big-bang explosion as the temperature goes down below some critical temperature as predicted by Grand Unified Theories (Zel'dovich et al [2]; Kibble [3, 4]; Everett[5], Vilenkin [6]).It is believed that cosmic strings give rise to density perturbations leading to the formation of galaxies (Zel'dovich [7]). These cosmic strings have stress energy and couple to the gravitational field for which it is interesting to study the effects of gravitation that 
arise from strings. So Letelier [8] and Stachel [9] are the authors who had started the general relativistic treatment of strings.

Moreover, electromagnetic field which contains highly ionized matter plays a key role for description of the energy distribution in the universe. String magnetic fields may be created due to adiabatic compression in cluster of galaxies and cosmic anisotropies may be attributed to the large scale magnetic fields. It is believed that in anisotropic models, the presence of electromagnetic field can alter the rate of creation of the particles and directly affects the rate of expansion of the universe.

The plane symmetric solution of basic gravitational equations have interesting applications in cosmology. Plane symmetric space-times with perfect fluid as the source, have been studied in general relativity owing to possible applications to astrophysics, cosmology and special relativistic hydrodynamics. It is well known that the matter in the actual universe obeys the perfect fluid hypothesis. Hence a large number of investigations in relativistic cosmology pertain to situations of perfect fluid distributions. The limiting case of equation of state $p=\rho$ has late been considered seriously by various authors. These fluids are known in the literature as stiff fluids or Zel'dovich fluids. The relevance of the equation of state "pressure = mass density" with regard to matter content of the universe has been brought to lime light by many authors. This equation of state is consistent with the limiting velocity of sound waves equal to the velocity of light. The solutions of the Einstein's equations with the "stiff equation" of state i.e., $p=\rho$ has been considered in several contexts, mostly of an astrophysical nature, particularly, in situations involving "white holes" lagging cores of a Big-bang cosmology, rotating neutron stars (pulsars) or a central region of quasars and inhomogeneous cosmological models.

Very few literatures are available on special barotropic fluid, with equation of state $p+\rho=0, \rho>0$. The fluid satisfying such an equation of state is known as " $\rho$ vacuum" or "degenerate vacuum" or "false vacuum" [ Blome and Priester[10], and Kaiser and Stebbins, [11].

The fluid satisfying the equation of state $p=0, \rho \neq 0$ and $\rho=3 p$ are respectively known as dust distribution and disorder distribution. Rao and Singh [12] have found exact solutions for a non-static spherical symmetric metric for above two cases. In the dust distribution, $\mathrm{p}=0$ has been used for cosmological purpose. During condensed phase of the evolution the concept of dust as well as the fluid breaks down (Bichteler [13]). Thus in this case, the quantum mechanical description has to be used. When pressure and energy density are zero i.e. $p=0=\rho$ then the case is known as vacuum case. In this case the space time does not contain any matter i.e., Tij $=0$.

Rosen[14-15], Yilmaz[16], Karade and Dhoble[17], Karade[18], Israelit[19-21], Liebscher[22], Reddy and Venkateswaralu[23], Deo and Thengane[24], Sahoo[25] and Mohanty et al.[26],Katore et al[27] ,Sahoo and Mishra[28,29] and Sahoo et al.[30] are some of the authors, who have studied various aspects of bimetric theory in different angles. In spite of the fact that many good works have been done, it is evident from literature that there is need for further investigation which may unravel some of the hidden secrets of the theory.

We know that Mohanty and Sahoo [31] have considered the problem of non-static plane symmetric meson field and mesonic perfect fluid in bimetric theory and found the cosmological model in 1st case where the scalar field becomes constant. However in later case they have shown that the bimetric theory does not admit perfect fluid but allows only mesonic scalar field where the scalar field is also constant. Moreover, non-static plane symmetric cosmological models are investigated by Sahu et al.[32] in Rosen's bimetric theory of gravitation in the context of cosmic string coupled with electromagnetic field, perfect fluid coupled with electromagnetic field and mesonic perfect fluid coupled with electromagnetic field. It is observed that the plane symmetric non-static cosmological model exists in 1st case but vacuum and false vacuum models are established in 2 nd case. The result found in 3rd case leads to the result already obtained and studied by Mohanty and Sahoo [31]. It is interesting to note that there is no contribution to electromagnetic field in both 2 nd and 3rd case. S

To the best of our knowledge no author has studied the non-static plane symmetric space-time in the context of cosmic strings coupled with electromagnetic field and perfect fluid. Therefore, in this paper we are interested to study this problem and this work will be an extension to the work already done by Mohanty and Sahoo [31] and Sahu et al.[32] .

\section{Metric and Rosen's field equations}

The plane symmetric non-static metric in the general form is

$\mathrm{ds} 2=\mathrm{e} 2 \mathrm{~h}(\mathrm{dt} 2-\mathrm{dr} 2-\mathrm{r} 2 \mathrm{~d} \theta 2-\mathrm{s} 2 \mathrm{dz} 2)$,

where $\mathrm{r}, \theta, \mathrm{z}$ are cylindrical polar co-ordinates and $\mathrm{h}, \mathrm{s}$ are functions of time ' $\mathrm{t}$ '.

As the metric (2) is Riemannian (non-flat), it's background flat space-time metric is

$\mathrm{d} \sigma 2=\mathrm{dt} 2-\mathrm{dr} 2-\mathrm{r} 2 \mathrm{~d} \theta 2-\mathrm{dz} 2$.

The energy momentum tensor for a cloud of massive string and perfect fluid distribution with electromagnetic field as studied by Latelier [8] and Stachel [9] is expressed as

$$
\mathrm{T}_{\mathrm{j}}^{\mathrm{i}}=(\mathrm{p}+\rho) u^{i} u_{j}-\mathrm{p} g_{j}^{i}-\lambda x^{i} x_{j}+\mathrm{E}_{\mathrm{j} \text { mag }}^{\mathrm{i}}
$$


together with

$u^{i} u_{i}=1=-x_{i} x^{i}$ and $u^{i} x_{i}=0$,

Where $\mathrm{p}$ is the isotropic pressure, $\rho$ is the rest energy density of the system of the string with massive particle attached to them, $\lambda$ is the tension density of the string and $E_{j}^{i}$ mag is the energy momentum tensor for magnetic field. According to Latelier [8], $\lambda$ be +ve or -ve , $u^{i}$ is the four-velocity vector and $x^{i}$ represents the anisotropic direction, i.e direction of the string.

We consider $\rho=\rho p+\lambda$,

where $\rho p$ is the particle energy density attached to the string.

The electromagnetic field $\mathrm{E}_{\mathrm{j} \text { mag }}^{\mathrm{i}}$ is given by

$E_{j \text { mag }}^{\mathrm{i}}=\mathrm{F}_{\mathrm{jr}} \mathrm{F}^{\mathrm{ir}}+\frac{1}{4} \mathrm{~F}_{\mathrm{ab}} \mathrm{F}^{\mathrm{ab}} \mathrm{g}_{\mathrm{j}}^{\mathrm{i}}$,

where $F_{i j}$ is the electromagnetic field tensor.

In the co-moving co-ordinate system taking the electromagnetic field along $\mathrm{X}$-axis, so that the only nonvanishing component of electromagnetic field tensor $F_{i j}$ is $F_{23}$.

The first set of Maxwell's equation

$$
\mathrm{F}_{[\mathrm{ij}, \mathrm{k}]}=\mathrm{F}_{\mathrm{ij}, \mathrm{k}}+\mathrm{F}_{\mathrm{jk}, \mathrm{i}}+\mathrm{F}_{\mathrm{ki}, \mathrm{j}}=0
$$

leads to the result $F_{23}=$ a constant $=\mathrm{L}$ (say).

Due to the assumption of infinite electrical conductivity by Maartens[33], we have $F_{14}=F_{24}=F_{34}=0$.The only non- vanishing component of $\mathrm{Fij}$ is $\mathrm{F}_{23}$.

From eqn.(7), the non- vanishing components of Eij corresponding to line element (2) are

$E_{1 \mathrm{mag}}^{1}=-E_{2 \mathrm{mag}}^{2}=-E_{3 \mathrm{mag}}^{3}=E_{4 \mathrm{mag}}^{4}=\frac{L^{2}}{2 s^{2} e^{4 h}}=\zeta($ say $)$.

where $\frac{L^{2}}{2}$ represents the magnetic field.

Here the four-velocity flow vector $u^{i}$ satisfies the condition

$$
\mathrm{g}_{\mathrm{ij}} \mathrm{u}^{\mathrm{i}} \mathrm{u}^{\mathrm{j}}=1 \text {. }
$$

Using equation (8), the non-vanishing components of energy momentum tensor $\mathrm{T}_{\mathrm{j}}^{\mathrm{i}}$ yield

$T_{1}^{1}=\lambda-\mathrm{p}+\zeta, T_{2}^{2}=T_{3}^{3}=-\mathrm{p}-\zeta$ and $T_{4}^{4}=\rho+\zeta$.

In co-moving coordinate system, Rosen's field equations (1) for the metric (2) and (3) with the help of (10) are found as

$$
\begin{aligned}
& \left(\frac{s_{4}}{s}\right)_{4}+2 h_{44}=16 \pi \mathrm{k}(\lambda-\mathrm{p}+\zeta), \\
& \left(\frac{s_{4}}{s}\right)_{4}+2 h_{44}=-16 \pi \mathrm{k}(\mathrm{p}+\zeta), \\
& \left(\frac{s_{4}}{s}\right)_{4}-2 h_{44}=16 \pi \mathrm{k}(\mathrm{p}+\zeta) \\
& \text { and } \quad\left(\frac{s_{4}}{s}\right)_{4}+2 h_{44}=16 \pi \mathrm{k}(\rho+\zeta) .
\end{aligned}
$$

\section{Solution of field equations}

From equations (12) and (13), one can get

$$
\left(\frac{s_{4}}{s}\right)_{4}=0
$$

Integrating (15), we have

$$
\mathrm{s}=e^{a_{1} t+a_{2}}
$$

where $a_{1}$ and $a_{2}$ are arbitrary constants and $a_{1} \neq 0$.

On substitution of equation (15) in equations (11) to (14), we have

$$
\lambda=\rho+p=-2 \zeta
$$

and

$$
h_{44}=-8 \pi \mathrm{k}(\mathrm{p}+\zeta) \text {. }
$$

Since the field equation system (11) to (14) is an under determined system with four eqns. in five unknowns i.e, s, h, p, $\rho$ and $\lambda$, we need one extra relation to obtain explicit solutions. For the deterministic solutions, we assume two conditions i.e first the universe is filled with barotropic perfect fluid so that pressure ' $p$ ' depend on energy density ' $\rho$ ' and vice versa. Hence we take the relation:

$$
\mathrm{p}=€ 1 \rho \text {, where }-1 \leq € 1 \leq 1 \text {. }
$$

Now assigning different value to $€ 1$, we can get different solutions.

Case-1: $\quad$ Suppose $€ 1=0$ and $\rho>0$.

In this case eqn.(19) reduces to

$$
\mathrm{p}=0 \text {. (Dust distribution) }
$$


Substituting the value of $\zeta$ from (8), and the value of $\mathrm{k}=\sqrt{\frac{\mathrm{g}}{\gamma}}=s . e^{4 h}$ in eqn. (18) using eqn.(16) and then integrating, we get

$\mathrm{h}=a_{3} \cdot e^{-\left(a_{1} t+a_{2}\right)}+a_{4} \mathrm{t}+a_{5}$,

where $a_{3}, a_{4}$ and $a_{5}$ are arbitrary constants and $a_{3}=\frac{-4 \pi L^{2}}{a_{1}^{2}}$ and $a_{4} \neq 0$.

Using eqns. (21), (8) and (16), eqn. (17) yields

$$
\lambda=-2 \zeta=-L^{2} e^{-\left[4 a_{3} \cdot e^{-\left(a_{1} t+a_{2}\right)}+\left(4 a_{4}+2 \mathrm{a}_{1}\right) \mathrm{t}+\left(4 a_{5}+2 a_{2}\right)\right]} .
$$

Use of eqn. (20), eqn. (17) yields

$$
\lambda=\rho \text {. }
$$

Hence corresponding to solutions given in (16) and (20) to (23), the space-time (2) can be written as $\mathrm{ds} 2=e^{2\left[a_{3} \cdot e^{-\left(a_{1} t+a_{2}\right)}+a_{4} \mathrm{t}+a_{5}\right]}\left\{\mathrm{dt}^{2}-\mathrm{dr}^{2}-\mathrm{r}^{2} \mathrm{~d} \theta^{2}-\mathrm{e}^{2\left(a_{1} t+a_{2}\right)} \mathrm{dz}^{2}\right\}$,

The model found in (24) is the non-static plane symmetric massive geometric- string perfect fluid model. This model is free from singularity at $\mathrm{t}=0$.

Case-II: $\quad$ Suppose $€ 1=-1$ and $\rho>0$. In this case eqn. (19) reduces to

$$
\mathrm{p}=-\rho . \quad \text { (False vacuum model) }
$$

Use of $p=-\rho$ in eqn.(17), we get $\lambda=0$.

Therefore, the non-static plane symmetric massive string magnetized false-vacuum cosmological model does not exit in bimetric theory of gravitation.

Case-III: Suppose $€ 1=1$ and $\rho>0$.In this case eqn.(19) reduces to

$$
\mathrm{p}=\rho . \quad \text { (Stiff fluid model) }
$$

Using eqns.(16) and (26) in eqn.(17), we obtain

$\frac{\lambda}{2}=\mathrm{p}=\rho=-\zeta=\frac{-L^{2}}{2 s^{2} e^{4 h}}=\frac{-L^{2}}{2} \cdot e^{-2\left(a_{1} t+a_{2}\right)-4 \mathrm{~h}}$

Again use of eqn.(27) in eqn.(18), we found that

$h_{44}=0$.

On integration eqn.(28) yields to

$$
\mathrm{h}=\left(b_{1} t+b_{2}\right)
$$

where $b_{1}, b_{2}$ are constants of integration and $b_{1} \neq 0$.

Now applying eqn. (29) in eqn. (27), we find

$\frac{\lambda}{2}=\mathrm{p}=\rho=\frac{-L^{2}}{2} \cdot e^{-2\left(a_{1} t+a_{2}\right)-4\left(b_{1} t+b_{2}\right)}=\frac{-L^{2}}{2} \cdot e^{\left(c_{1} t+c_{2}\right)}$.
where $\quad c_{1}=-2\left(a_{1}+2 b_{1}\right)$ and $c_{2}=-2\left(a_{2}+2 b_{2}\right)$.

From eqn. (30), we have

$$
\lambda=-L^{2} \cdot e^{\left(c_{1} t+c_{2}\right)} \text {. }
$$

Hence the non-static plane symmetric massive string magnetized stiff fluid cosmological model in bimetric theory becomes

$\mathrm{ds} 2=e^{2\left(b_{1} t+b_{2}\right)} \cdot\left\{\mathrm{dt}^{2}-\mathrm{dr}^{2}-\mathrm{r}^{2} \mathrm{~d} \theta^{2}-\mathrm{e}^{2\left(a_{1} t+a_{2}\right)} \mathrm{dz}^{2}\right\}$.

It is interesting that the model (32) is singularity free at $\mathrm{t}=0$.

Case-IV: Suppose $p=\rho=0$ (Vacuum model).

On substitution $p=\rho=0$ in eqn.(17), one can get $\lambda=0$.

Hence the non-static plane symmetric massive string magnetized vacuum cosmological model does not sustain in bimetric theory.

\section{Physical Properties of the models :}

4.1 The kinematical parameters of the model (24) are as follows:

The spatial volume $\mathrm{V}$ of the universe for the model (24) is found to be

$$
\mathrm{V}=\sqrt{-g}=e^{\left[4 a_{3} \cdot e^{-\left(a_{1} t+a_{2}\right)}+\left(4 a_{4}+\mathrm{a}_{1}\right) \mathrm{t}+\left(4 a_{5}+a_{2}\right)\right]} \text {. }
$$

Now $\mathrm{V} \rightarrow$ constant as $\mathrm{t} \rightarrow 0$ and $\mathrm{V} \rightarrow \infty$ as $\mathrm{t} \rightarrow \infty$. Thus the volume of universe increases with respect to increase of time. Hence it is inferred that the model starts with a constant volume and blows up at infinite future.

The Expansion Scalar $\theta$ for the model (24) is found as

$$
\theta=u_{; i}^{i}=-e^{-\left(a_{3} \cdot e^{-\left(a_{1} t+a_{2}\right)}+a_{4} \mathrm{t}+a_{5}\right)}\left\{3 a_{1} a_{3} e^{-\left(a_{1} t+a_{2}\right)}-\left(3 a_{4}+\mathrm{a}_{1}\right)\right\} .
$$

When $\mathrm{t} \rightarrow 0, \theta \rightarrow$ constant and as $\mathrm{t} \rightarrow \infty, \theta \rightarrow 0$. Hence it is inferred that the rate of expansion of the universe at initial epoch is constant. However the expansion becomes very slow as time increases and there will be no expansion at infinite future.

The shear scalar $\sigma$ defined by Ray Choudhuri [ 34 ] as 
$\sigma 2=\frac{1}{12}\left\{\left[\frac{g_{11,4}}{g_{11}}-\frac{g_{22,4}}{g_{22}}\right]^{2}+\left[\frac{g_{22,4}}{g_{22}}-\frac{g_{33,4}}{g_{33}}\right]^{2}+\left[\frac{g_{33,4}}{g_{33}}-\frac{g_{11,4}}{g_{11}}\right]^{2}\right\}$.

Thus the anisotropy of the universe is found to be

$$
\begin{aligned}
& \sigma=\sqrt{\frac{2}{3}}\left(a_{1}\right)=(\text { constant }) . \\
& \text { and } \frac{\sigma}{\theta}=-\frac{\sqrt{\frac{2}{3}} a_{1} e^{a_{3} \cdot e^{-\left(a_{1} t+a_{2}\right)}+a_{4} \mathrm{t}+a_{5}}}{3 a_{1} a_{3} e^{-\left(a_{1} t+a_{2}\right)}-\left(3 a_{4}+a_{1}\right)} \text {. } \\
& \text { Now } \frac{\sigma}{\theta} \rightarrow \text { constant as } \mathrm{t} \rightarrow 0 \text { and } \frac{\sigma}{\theta} \rightarrow \infty \text { as } \mathrm{t} \rightarrow \infty \text {. }
\end{aligned}
$$

The shear scalar $\sigma$ is constant throughout the evolution of the universe. Hence this result indicates that the model is anisotropic in nature at the initial time and will continue throughout the evolution.

The physical parameters such as pressure $p$ and particle energy density $\rho p$ are found in the model as

$$
\rho p=\rho-\lambda=0=p \text {. }
$$

So when $\mathrm{t} \rightarrow 0, \lambda(=\rho) \rightarrow \mathrm{a}$ constant and as $\mathrm{t} \rightarrow \infty, \lambda(=\rho) \rightarrow 0$. Hence the model reduces to an empty universe.

4.2. The kinematical parameters of the model (32) are as follows:

The spatial volume $\mathrm{V}$ of the universe for the model (32) is found to be

$$
\mathrm{V}=\sqrt{-g}=e^{\left[d_{1} t+d_{2}\right]}
$$

where $\quad d_{1}=\left(a_{1}+4 b_{1}\right)$ and $d_{2}=\left(a_{2}+4 b_{2}\right)$.

From above it is observed that as $\mathrm{t} \rightarrow 0, \mathrm{~V} \rightarrow \mathrm{a}$ constant and $\mathrm{V} \rightarrow \infty$ as $\mathrm{t} \rightarrow \infty$. Thus the volume of universe increases with increase of time. Hence it is inferred that the model starts with a constant volume and blows up at infinite future.

The Expansion Scalar $\theta$ for the model (32) is found as

$$
\theta=u_{; i}^{i}=-\mathrm{d}_{3} \cdot e^{-\left(b_{1} t+b_{2}\right)} \quad, \text { where } \mathrm{d}_{3}=3 \mathrm{~b}_{1}+\mathrm{a}_{1}
$$

When $\mathrm{t} \rightarrow 0, \theta \rightarrow$ constant and as $\mathrm{t} \rightarrow \infty, \theta \rightarrow 0$. Hence it is inferred that the rate of expansion of the universe at initial time is constant. However, the expansion becomes very slow as time increases and there will be no expansion at infinite future.

The shear scalar $\sigma$ (anisotropy of the universe )as defined as in case -4.1 is found to be

$\sigma=\sqrt{\frac{2}{3}}\left(a_{1}\right)=($ constant $) \quad$ and $\quad \frac{\sigma}{\theta}=\frac{\sqrt{\frac{2}{3}\left(a_{1}\right)}}{\mathrm{d}_{3}} \cdot e^{\left(b_{1} t+b_{2}\right)}$.

Now $\frac{\sigma}{\theta} \rightarrow$ constant as $\mathrm{t} \rightarrow 0$ and $\quad \frac{\sigma}{\theta} \rightarrow \infty$ as $\mathrm{t} \rightarrow \infty$.

As the shear scalar $\sigma$ is constant at the initial time and at infinite future, the model is anisotropic in nature throughout the evolution.

The physical parameters involved in the model (32) are found as

$$
\begin{aligned}
& \mathrm{p}=\rho=\frac{-L^{2}}{2} \cdot e^{\left(c_{1} t+c_{2}\right)}, \quad \lambda=-L^{2} \cdot e^{\left(c_{1} t+c_{2}\right)} \\
& \text { and } \rho \mathrm{p}=\rho-\lambda=\frac{L^{2}}{2} \cdot e^{\left(c_{1} t+c_{2}\right)} .
\end{aligned}
$$

From above data it is seen that as time increases $\rho p$ increases but other parameters decreases.

\section{Conclusion}

The present work reported in this paper can be considered as an extension to the work of Mohanty and Sahoo [31] and Sahu et al.[32]. In view of recent interest of readers in electromagnetic field in free space we took an attempt to develop the idea of perfect fluid and cosmic string considering non-static plane symmetric cosmological model in bimetric theory of gravitation. The model obtained in case- 1 is a geometric string model and in this model the universe starts from a constant volume and blows up at infinite future. Also the model has anisotropic in nature at the beginning and also throughout the evolution. The rate of expansion in the model gradually decreases as time increases and there will be no expansion at infinite time. We also see that the there no effect of magnetic field in the expansion of the universe and the parameters remain finite and physically significant for all $t>0$.It is observed that there is no contribution to electromagnetic field in both second and third case. However, vacuum model and false vacuum model are established in second case. In the case of false vacuum model, it is seen that the rate of expansion of the universe is increasing and the universe will collapse at infinite future. It is also observed that the models found are singularities free.

\section{References}

[2]. Zel'dovich,Ya. B.; Kobzarev, I.yu., Okun, L.B..: Sov. Phys.JETP 40,1(1975).

[3]. Kibble,T.W.B.: J.Phys.A.Math.Gen.9,1387 (1976).

[4]. Kibble, T.W.B.: Phys.Rep.67,183(1980). 
[5]. Everett, A.E.: Phys.Rev.24,858(1981).

[6]. Vilenkin, A.: Phys.Rev. D24, 2082(1981).

[7]. Zel'dovich, Ya. B.: Mon.Not.R.Astron.Soc.192, 663(1980).

[8]. Letelier, P.S. : Phys. Rev.D.; 28,2414(1983).

[9]. Stachel, J. : Phys. Rev.D.; 21,2171(1980).

[10]. Blome, J.J. and Priester,W.: Naturewissenshaften,71,528(1984).

[11]. Kaiser, N. and Stebbins, A.: Nature,310, 391(1984).

[12]. Rao, J.R. and Singh, R.K.T.: Appeared in Proc. Einstein Centenary Symposium, Nagpur(1980).

[13]. Bichteler, K. : Comm.Math.Phy. 4, 352(1967).

[14]. Rosen, N. : Gen. Relativ.Grav.; 6,259(1975).

[15]. Rosen, N. : Gen. Relativ.Grav.; 9,339(1978).

[16]. Yilmaz, H. : Gen. Relativ.Grav.; 6,269(1975)

[17]. Karade, T.M.; Dhoble, Y.S. : Lett.Nuovo Cimento.; 29,390(1980).

[18]. Karade, T.M. : Indian J.Pure Appl. Math.; 11,1202(1980).

[19]. Israelit, M. : Gen. Relativ.Grav.; 7,623(1976).

[20]. Israelit, M. : Gen. Relativ.Grav.; 11,25(1979).

[21]. Israelit, M. : Gen. Relativ.Grav.; 13,681(1981).

[22]. Liebscher, D.E.: Gen. Relativ.Grav. ; 6,277(1975).

[23]. Reddy, D.R.K.; Venkateswaralu, R.: Astrophy. Space Sci.; 259,169(1989).

[24]. Deo, S. ; Thengane, K.D. : FIZIKAB II; 3,155(2002).

[25]. Sahoo, P.K.: Int. J. Theor. Phy. DOI 10.1007/s 10773-009-0174-Z(2009).

[26]. Mohanty,G.; Sahoo,P.K.and Mishra,B : Astrophy. Space Sci.; 281,609(2002).

[27]. Katore, S.D.; Rane, R.S. and Wankhade, K.S.: Pramana-J. Phys. ;76, No.-4(2011).

[28]. Sahoo ,P.K.and Mishra, B. : Int. Jour. Pure and App. Math., 93(2) 275-284 (2014).

[29]. Sahoo, P.K.and Mishra.B : Astrophy. Space Sci. 349(1), 491-499 (2014).

[30]. Sahoo,P.K.; Mishra, B and G. Chakradhar Reddy : The Euro. Phy. J. Plus, 129, 49 (2014).

[31]. Mohanty,G., Sahoo, P.K. : Czech. J.Phy.; 52,1041(2002).

[32]. Sahu ,R.C. ; Patra,R.N.; Behera,B.; Nayak, B.K.: The International Journal of Science and Technoledge 2, 164(2014).

[33]. Maartens, R.: Pramana-J.Phys.55, 576(2000)

[34]. Ray Chaudhuri, A.K. : Phys. Rev.; 98, Issue 4, 1123 (1955). 